\title{
25 Research Soure \\ Plexciton Modes Guided by an Exciton Slab in a Columnar Thin Film
}

\author{
Mona Rostami \\ University of Qom \\ Ferydon Babaei ( $\nabla$ fbabaei@qom.ac.ir) \\ university of qom https://orcid.org/0000-0003-1704-0474
}

\section{Research Article}

Keywords: plexciton, Rabi splitting, columnar thin film, transfer matrix method

Posted Date: December 8th, 2021

DOI: https://doi.org/10.21203/rs.3.rs-1039230/v1

License: (c) (i) This work is licensed under a Creative Commons Attribution 4.0 International License. Read Full License 


\section{Abstract}

In this study, we reported plasmon-exciton coupling for excitation the surface plexciton in columnar thin film with a central exciton slab using the transfer matrix method in Kretschmann configuration. The optical absorption spectra for surface plasmon polariton, surface exciton and surface plexciton was investigated at different structural parameters in proposed structure. The characteristics of surface optical modes were analyzed and there was an anticrossing behavior between polariton branches of plexciton spectra. Localization of surface modes on interfaces and hybridization between plasmons and excitons at both interfaces of exciton slab were proved by the time-averaged Poynting vector. We found that the types of coupling regimes between plasmons and excitons from weak to strong could be achieved. We found a high Rabi splitting energy $840 \mathrm{meV}$ corresponding to the time period $5 \mathrm{fs}$ which includes to the fast energy transfer between surface plasmon polaritons and surface excitons.

\section{Introduction}

The plexciton modes are new optical hybrid modes that result from a combination of exciton mode and plasmon polariton mode. Polariton modes usually split into two branches when the plasmon polariton frequency is close to the exciton frequency. In a plexcitonic system, the energy transfer between plasmons and excitons is of particular importance. Design a plexcitonic device with ultrafast energy transfer could be a mechanism for switching light by light on the nanoscale [1]. This energy transfer depends on the strength of the coupling between plasmons and excitons. The fast energy transfer can occur in a strong coupling regime with large Rabi splitting energy [2, 3]. If the energies of the new optical modes are plotted as a function of the detuning energy (the energy difference between the plasmon and the exciton peaks), an anticrossing behavior is seen in the zero detuning with a minimum energy separation called the Rabi splitting energy, which determines the strength of the coupling between plasmons and excitons [4]. In the strong coupling regime, the excitation energy is shared and oscillates between plasmons and excitons (Rabi oscillations) $[5,6]$. Some of the applications of the strong coupling between plasmons and excitons have been demonstrated, including Fabry-Perot cavities [7], photonic crystals [8], semiconductor microcavities [9, 10], single-atom lasers [11], all-optical switches [12, 13] and quantum information processing [14].

In this study, the plasmon-exciton coupling in a columnar thin film with central exciton slab was theoretically investigated under Kretschmann configuration using the transfer matrix method. In our work, the plexcitonic system consisted of a plasmonic medium (Silver thin film) and a trilayer structure (Magnesium fluoride columnar thin film-J aggregate slab- Magnesium fluoride columnar thin film). We considered the excitons (Frankel excitons at room temperature) of a cyanine dye [5,5区,6,6囚-tetrachloro-di(4-sulfobutyl) benzimidazolocarbocyanine(TDBC molecule) were self-assembled as J-aggregate due to strong dipole moment [15]. The theory in brief is presented in Section II, and followed by the results and discussions in Section III.

\section{li. Theory In Brief}


Let us, consider a Kretschmann configuration according to Fig. 1 for coupling surface plasmon polariton and exciton. The region $0 \leq z \leq d_{1}$ is considered by a silver metal thin film with relative permittivity $\epsilon_{A g}$. The region $d_{1} \leq z \leq\left(d_{1}+d_{2}\right)$ is occupied by an oblique $\mathrm{MgF}_{2}$ columnar thin film, where $d_{2}=L \sin \chi$, $L$ and $\chi$ is length and tilt angle of nanocolumns of columnar thin film, respectively. In addition, the region $\left(d_{1}+d_{2}\right) \leq z \leq\left(d_{1}+d_{2}+d_{3}\right)$ is considered by an exciton slab (J-aggregate dyes) with relative permittivity $\epsilon_{j a}$. Again, the region $\left(d_{1}+d_{2}+d_{3}\right) \leq z \leq\left(d_{t}=d_{1}+2 d_{2}+d_{3}\right)$ is occupied by oblique $\mathrm{MgF}_{2}$ columnar thin film, while the regions $z \leq 0$ and $z \geq d_{t}$ are respectively a prism with relative permittivity $\epsilon_{1}=n_{1}^{2}$ and an air medium $\left(\mathrm{n}_{2}=1\right)$. The anisotropic dielectric permittivity tensor $\epsilon_{M g F_{2}}$ for oblique $\mathrm{MgF}_{2}$ columnar thin film(CTF) is defined as:

$\epsilon_{M g F_{2}}=S_{y}(\chi) \cdot \epsilon^{r e f} \cdot S_{y}^{T}(\chi)(1)$,

where the superscript $T$ indicates to the transpose of a tensor and $\chi$ is tilt angle of columns. The reference relative permittivity and tilt rotation tensors are respectively as:

$\epsilon^{r e f}=\epsilon_{a} u_{z} u_{z}+\epsilon_{b} u_{x} u_{x}+\epsilon_{c} u_{y} u_{y}(2)$,

$S_{y}(\chi)=\left(u_{x} u_{x}+u_{z} u_{z}\right) \cos \chi+\left(u_{z} u_{x}-u_{x} u_{x}\right) \sin \chi+u_{y} u_{y}(3)$,

where $\epsilon_{a, b, c}$ and $u_{x, y, z}$ are the effective relative permittivity scalars of the oblique $\mathrm{MgF}_{2}$ columnar thin film and the Cartesian unit vectors, respectively. The $\epsilon_{a, b, c}$ could be obtained from the Bruggeman homogenization formalism [16].

The considered structure (Fig. 1) exposed by p-linear polarized plane wave from the bottom of the prism at an angle to the $z$ - axis in xz-plane. We can calculate the reflectance $\left(r_{i=s, p}\right)$ and transmittance $\left(t_{i=s, p}\right.$ ) amplitude using both Maxwell's curl equations, the continuity of the tangential components of electrical and magnetic fields at interfaces and solving following the algebraic matrix equation [17]:

$\left\{\backslash \text { begin }\{\text { array }\}\{c c\}\left(\{t\}_{-}\{s\} \&\{t\}_{-}\{p\} \backslash \text { end }\{\text { array }\} 00\right)\right\}^{\wedge}\{T\}=\left\{K \backslash \text { left }\left(\backslash \text { stackrel }\{\}^{\prime}\{\text { theta }\} \backslash \text { right }\right)\right\}^{\wedge}\{-1\}$.

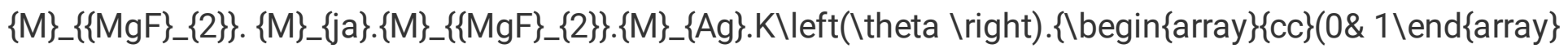
$\left.\{r\}\left\{\{s\}\{r\}_{-}\{p\}\right)\right\}^{\wedge}\{T\}(4)$,

all computational details in Eq. 4 are contained in published literatures[18-21],where $\left(r_{s}, r_{p}\right)$ and $\left(t_{s}, t_{p}\right)$ are the amplitudes of reflected and transmitted waves with $s$ - and $p$-linear polarizations. Then, we can obtain optical absorption as $A=1-\{\mid \text { sum }\}_{-}\{i=s, p\}\left(\{R\}_{-}\{i p\}+\{T\}_{-}\{i p\}\right)$, where $\left.\{R\}_{-}\{i p\}=\left\{L\{r\}_{-}\{i\}\right]\right\}^{\wedge}\{2\}$ and $\{T\}_{-}\{i p\}=\backslash$ left $(\backslash \operatorname{left}(\operatorname{Re} \backslash$ left$[\backslash$ text $\{\cos \} \backslash$ left $(\backslash$ stackrel \{\}$\backslash \backslash$ theta $\} \backslash$ right $) \backslash$ right $] \backslash$ right $) /\left(\{\mathrm{n}\}_{-}\{1\}\right.$ $\backslash$ text $\{c\} \backslash \operatorname{text}\{0\} \backslash \operatorname{text}\{s\}(\backslash$ theta $) \backslash$ right $)\left\{[\{t\}\{\{i\}]\}^{\wedge}\{2\}\right.$.

\section{lii. Results And Discussions}


Figure 2 shows absorption density plots as functions of wavelength and incident angle of $\mathrm{p}$-linear polarization light plane wave for excitation of surface plasmon polariton (SPP), surface exciton(X) and surface plexciton (PLX). The fixed parameters in optical modeling were $d_{1}=40 \mathrm{~nm}, \mathrm{~L}=600 \mathrm{~nm}, \mathrm{~d}_{3}=286$ $n m, n_{1}=2.57, \chi=20^{\circ}, f_{v}=0.8, \theta_{\delta}=25.5^{\circ}$, where $f_{v}$ is porosity of columnar thin film. The detuning frequency is introduced as $\backslash$ delta $=\{\text { \omega }\}_{-}\{$spp $\}\{\text {\lomega }\}_{-}\{x\}$ so that the zero detuning frequency ( $($ delta $=0)$ is specified by a vertical line in each of plots. The $\{\backslash \text { theta }\}_{-}\{$\delta $\}$(we call this the detuning angle) indicates to the angular position of incident light where the detuning frequency is zero (\{lomega \}$_{-}\{$spp $\}=\{\text {\omega }\}_{-}\{x\}$ ) which is marked with a horizontal line in Fig. 2c. Inset plots show the optical absorption as a function of wavelength at detuning angle. We scanned the wavelength and the incident angle with increment $1 \mathrm{~nm}$ and $0.01^{\circ}$, respectively. Although, in an experiment work, reaching to the zero detuning frequency is difficult due to the limitations of measuring instruments. It must be mentioned here, in depiction each of the density plots for bare SPP and bare X, the thickness of metal and exciton slab was zero, respectively (see Figs. 2a \& Figs. 2b). It is observed in Fig. 2c that when frequency of the plasmon sates equals to frequency of the exciton states, the polariton mode in a plexciton system is splits to two branches upper polariton (UP) and lower polariton (LP). It is clear that there exist an anticrossing behavior between UP and LP polariton branches at zero detuning frequency. This matter is consistent with the coupled oscillator model (COM) [22] that it express, when two or more oscillators with specific resonant frequencies are coupled, new hybrid resonant frequencies appear.

The absolute value of Cartesian components of electric and magnetic fields along the z-axis are given in Fig. 3 at $\lambda=594 \mathrm{~nm}$ with the same values used in Fig. 2 for the parameters. It is found in Fig. 3a1 \& Fig. 3a2 that the intensity of the fields $\backslash$ left $\mid\{E\}_{-}\{x, z\} \backslash$ right| and $\backslash$ left $\mid\{H\}_{-}\{y\} \backslash$ right| has increased at interface of metal and columnar thin film $(z=40 \mathrm{~nm})$. These fields are damped away from the interface, which it indicates to excitation and localization of surface plasmon polariton wave at metal/CTF interface. Slight roughness in the fields at $z=245 \mathrm{~nm}$ is related to the removal of exciton slab in structure to propagate the surface plasmon polariton wave, which creates this numerical discontinuity in the calculations. Because we used only one code and this issue could be solved by considering a CTF with length $2 \mathrm{~L}$ instead of two CTFs of upper and lower each of length $\mathrm{L}$. In order to surface exciton wave, the metal thickness was considered zero in structure, see Fig. 3b1\& Fig. 3b2. The $z=205 \mathrm{~nm}$ and $z=491 \mathrm{~nm}$ refer to CTF/exciton slab (lower interface) and exciton slab/CTF (upper interface), respectively. It is seen that the exciton surface wave is excited at both interfaces of exciton slab. We repeated the same calculations for the whole structure at $\lambda=594 \mathrm{~nm}\left(\{\text { \omega }\}_{-}\{\mathrm{spp}\}=\{\text { lomega }\}_{-}\{x\}\right)$ as shown in Fig. $3 \mathrm{c} 1 \&$ Fig. 3c2. It is observed that the intensity of electromagnetic fields is decreased at metal/CTF interface $(z=40 \mathrm{~nm})$ and we expect this energy to be transferred to the excitons and a coupling to take place at both interfaces of exciton slab.

In order to further analyzation the coupling between plasmons and excitons, the Cartesian components of time-averaged Poynting vector and local absorption (LAP) [20] as a function of the depth of the structure are shown in Fig. 4. The increase of the $\mathrm{x}$ - component Poynting vector compared to the other components indicates to the localization of the surface plasmon polariton wave at the metal/CTF interface (Fig. 4a1). 
It is well known in local absorption plot (Fig. 4a2) with maximum intensity at $z=40 \mathrm{~nm}$. The Fig. 4b1\&Fig. 4b2 show the excitation of surface exciton wave at both interface of exciton slab. The surface plexciton waves are excited at internal (lower surface) and external interfaces (upper surface) of exciton slab (see Fig. 4c1\&Fig. 4c2). A comparison between the plots of the local absorption show the intensity of absorption at $z=40 \mathrm{~nm}$ is decreased in Fig. 4c2, while it increased at both interfaces of exciton slab. Then an energy transfer has occurred from surface plasmon polaritons to surface excitons. On the other hand, some of quasiparticles of SPPs have migrated to both interfaces of exciton slab where the surface excitons are present. Therefore, the hybridization between of them creates new optical quasiparticle called plexciton. The reason for the greater local absorption at external interface of exciton is due to the increase in the optical path of the photons. In the following, we discussed the characteristics of this hybridization between plasmons and excitons in terms of different structural parameters.

The energy of surface polariton modes as a function of detuning frequency for different refractive index of prism is shown in Figs. 5a1-5a3. The structure is described by the fixed parameters: $d_{1}=40 \mathrm{~nm}, L=600$ $n m, d_{3}=286 \mathrm{~nm}, X=20^{\circ}, f_{v}=0.8$. It is observed that the surface exciton mode $(X)$ is almost at $2.1 \mathrm{eV}$ and its value does not change with changing the detuning frequency and the refractive index of prism. The surface plasmon polariton mode (SPP) in terms of the detuning frequency increases linearly. The intersection of SPP and X modes indicates a zero detuning frequency ( $($ delta $=0)$. Two upper and lower surface plexciton branches in the rangeldelta $=\backslash$ left(- $\backslash$ text $\{0.2,0.2\} \backslash$ right $) \mathrm{eV}$ are clearly visible in plots. It is found that the separation distance of the branches of plexciton modes increased slightly by increasing the refractive index of the prism. The full width half maximum (FWHM) of plasmons $(0$, excitons () , Rabi splitting energy (\{\Omega $\})$ and detuning angle ( $\{\backslash \text { theta }\}_{-}(\{$delta $\})$as a function of the refractive index of prism are given in Figs. 5b\& Fig. 5c, respectively. The Rabi splitting energy in our work is calculated as

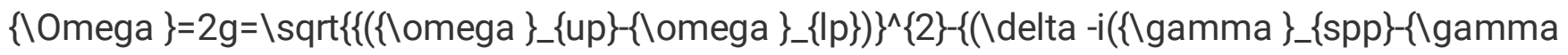
\}$\left.\left.\left.\left._{-}\{x\}\right) / 2\right)\right\}^{\wedge}\{2\}\right\}[23]$, where $g$ is the coupling energy, $\{\text { lomega }\}_{-}\{$up $\}$and $\{\text {lomega }\}_{-}\{\mid \mathrm{p}\}$ are respectively the energy of upper and lower polariton peaks in plexciton spectra. It is seen that the both SPP and X modes were increased slightly by increasing $\mathrm{n}_{1}$, while the detuning angle decreased and the Rabi splitting energy increased slightly. Herein, there is an intermediate coupling regime (ICR) ( $\{$ gamma \}$_{-}\{x\}$ cript $\left.>\right) \backslash$ left[24\right] between SPP and X modes. In ICR, the normal mode splitting occurs in the frequency domain, and an anticrossing behavior generally observed in optical absorption spectra [25].

The energy of SPP, $X$ and PLX modes as a function of detuning frequency for different thicknesses of plasmon medium is depicted in Figs. 6a1-6a3.All parameters are the same as Fig. 5,except that $n_{1}=2.57$. Similar to the Fig. 5, the same trend can be seen in the energy of the modes. Altogether, the upper and lower plexciton branches approach the plasmon mode at high and low detuning frequency, respectively. The values of , , \{\Omega $\}$ and $\{\backslash \text { theta }\}_{-}\{\backslash$ delta $\}$ as a function of the thicknesses of metal are shown in Figs. 6b\& Fig. 6c, respectively. Both of and $\{\backslash \text { theta }\}_{-}\{$\{delta $\}$are approximately constant in terms of the thickness of the plasmonic medium. However, and \{\Omega $\}$ decreased with increasing $d_{1}$. Here also, the coupling between SPP and X modes was ICR. The energy of polariton modes as a function of \delta at different L, damping of plasmons and excitons, Rabi splitting energy and detuning 
angle as a function length of nanocolumns of dielectric medium $\left(\mathrm{MgF}_{2}\right)$ are given in Figs. $7 \mathrm{a}-7 \mathrm{c}$. All parameters are the same as Fig. 6 , except that $d_{1}=40 \mathrm{~nm}$.It is clear that the SPP, $X$ and LP modes are almost degenerated at $L=200 \mathrm{~nm}$ while these modes are isolated at $L=600 \mathrm{~nm} \rrbracket$. Due to the high energy separation between plexciton branches and the close damping of plasmons and excitons, the Rabi splitting energy increased, while the detuning angle was almost constant. It is found that there exist a strong coupling regime (SCR) $\left(2 \mathrm{~g}>\left(\{\backslash \text { gamma }\}_{-}\{\mathrm{spp}\},\{\backslash \text { Igamma }\}_{-}\{\mathrm{x}\}\right)\right)$ between plasmons and excitons. In SCR, the energy can be coherently transferred between the two SPP and X modes for at least a few times before their eventual decay [24]. Here, Rabi splitting energy was between $430 \mathrm{meV}$ and $840 \mathrm{meV}$ which it corresponds to the the time period $T=2 \backslash$ pi $/\{\backslash$ Omega $\}$ for Rabi oscillation as much as 5-10 $\mathrm{fs}$. This time indicates to the fast energy transfer between surface plasmon polaritons and surface excitons.

The energy of surface polariton modes as a function of detuning frequency for different tilt angles of nanocolumns of dielectric medium is shown in Figs. 8a1-8a3 and also the damping of plasmons and excitons, splitting energy and detuning angle are given in Figs. 8b-8c, all parameters used in optical modeling were same as Fig. 7,excpet that $L=600 \mathrm{~nm}$. The general pattern in SPP and X modes is the same as before in terms of detuning frequency and the same anticrossing behavior was observed in plexciton modes. The values of , $\{\backslash$ Omega $\}$ decreased by increasing of $\chi$, and increased, while $\{\backslash$ theta \}$_{-}\{$Idelta $\}$was almost constant. The coupling between SPP and X modes was ICR. Next, we followed the same calculations for the effect of the porosity $\left(f_{v}\right)$ of the CTF on surface polariton modes as obtained results are depicted in Figs. 9a-9c. The fixed parameters in Fig .9 were $d_{1}=40 \mathrm{~nm}, \mathrm{~L}=600 \mathrm{~nm}, \mathrm{n}_{1}=2.57$. But, in order to see the surface polariton modes and the anticrossing behavior in PLX branches, we selected the thickness of exciton slab and tilt angle of nanocolumns of dielectric medium at void fractions of CTF $0.2,0.4,0.6$ and 0.8 respectively as: $15^{\circ}, 478 \mathrm{~nm} ; 20^{\circ}, 370 \mathrm{~nm} ; 20^{\circ}, 315 \mathrm{~nm}$ and $20^{\circ}, 286 \mathrm{~nm}$. It is observed that the, $\{\backslash$ Omega $\}$ and $\{\backslash \text { theta }\}_{-}\{\backslash$ delta $\}$ decreased by increasing porosity of CTF, while increased. The values of porosity change the effective refractive index of CTF and at low porosity, a denser CTF is obtained. We found here too an ICR between SPP and X modes. Finally, we repeated the same quantities as a function of thickness of exciton slab as shown in Figs. 10a-10c, all parameters were same as Fig. 9 except that $\chi=25^{\circ}, f_{v}=0.8$. In our work, the SPP,X and LP modes were degenerate at $d_{3}=225 \mathrm{~nm}$ while at other thicknesses of exciton slab were isolate. Herein, we found a SCR at $d_{3}=150,175 \mathrm{~nm}$ and obtained an ICR at else $d_{3}$. Therefore, different coupling regimes can be achieved by adjusting structural parameters in proposed structure. In this field, researchers are looking for high splitting energy, so we did not pursue the weak coupling regime, where the optical modes of the coupled system remain unchanged from their initial uncoupled states.

\section{Iv. Conclusion}

The coupling between surface plasmon polaritons and surface excitons was theoretically studied by a central exciton slab in columnar thin film in Kretschmann configuration using transfer matrix method. The surface polariton modes were extracted from optical absorption spectra and characteristics of them were investigated at different structural parameters. It is found that that different coupling regimes 
between plasmons and excitons can be achieved by adjusting structural parameters in proposed structure. More localization of the surface optical modes was proved by the components of the timeaveraged Poynting vector as a function of depth of the structure. The anticrossing behavior was observed between upper and lower polariton branches in plexciton system, while the SPP and X modes had same trend. It is obtained that the damping of plasmons and excitons reduced the Rabi splitting energy. In our work, we achieved to high Rabi splitting energy $840 \mathrm{meV}$ that it showed there is a strong coupling regime between SPP and $\mathrm{X}$ modes. Therefore, a fast energy transfer occurs from the order of $5 \mathrm{fs}$ between plasmons and excitons.

\section{Declarations}

\section{Acknowledgements}

This work was carried out with the support of the University of Qom.

\section{-Funding}

Not applicable.

\section{-Competing interests}

The authors declare no competing interests. The authors alone are responsible for the content and writing of this article.

\section{-Code availability}

Not applicable.

\section{-Authors' contributions}

Mona Rostami: Investigation, Writing original draft, Simulation, Program code running

Ferydon Babaei: Work idea, Writing, Simulation

\section{- Ethics approval}

All analyses were based on previous published studies, experimental and theoretical simulation, thus no ethical approval and patient consent are required.

\section{-Consent to participate}

Written informed consent for publication was obtained from all participants. The authors consented to participate. 


\section{-Consent for publication}

Written informed consent for publication was obtained from all participants. The authors consented to publish.

\section{Availability of date and materials}

All data generated or analyzed during this study are included in this published article.

\section{References}

[1] Vasa, P., Wang, W., Pomraenke, R., Lammers, M., Maiuri, M., Manzoni, C., Cerullo, G., \& Lienau, C. (2013). Real-time observation of ultrafast Rabi oscillations between excitons and plasmons in metal nanostructures with J-aggregates. Nature Photonics, 7(2), 128-132.

[2] Sukharev, M., Seideman, T., Gordon, R. J., Salomon, A., \& Prior, Y. (2014). Ultrafast energy transfer between molecular assemblies and surface plasmons in the strong coupling regime. Acs Nano, 8(1), 807817.

[3] Symonds, C., Bonnand, C., Plenet, J. C., Bréhier, A., Parashkov, R., Lauret, J. S., Deleporte, E.,\& Bellessa, J. (2008). Particularities of surface plasmon-exciton strong coupling with large Rabi splitting. New Journal of Physics, 10(6), 065017.

[4] Balci, S., Kucukoz, B., Balci, O., Karatay, A., Kocabas, C., \& Yaglioglu, G. (2016). Tunable plexcitonic nanoparticles: a model system for studying plasmon-exciton interaction from the weak to the ultrastrong coupling regime. Acs Photonics, 3(11), 2010-2016.

[5] Achermann, M. (2010). Exciton- plasmon interactions in metal- semiconductor nanostructures. The Journal of Physical Chemistry Letters, 1(19), 2837-2843.

[6] Sun, J., Li, Y., Hu, H., Chen, W., Zheng, D., Zhang, S., \& Xu, H. (2021). Strong plasmon-exciton coupling in transition metal dichalcogenides and plasmonic nanostructures. Nanoscale, 13(8), 4408-4419.

[7] Hood, C. J., Chapman, M. S., Lynn, T. W., \& Kimble, H. J. (1998). Real-time cavity QED with single atoms. Physical review letters, 80(19), 4157.

[8] Hennessy, K., Badolato, A., Winger, M., Gerace, D., Atature, M., Gulde, S., Falt, S.,Hu, E.L., \& Imamoglu, A. (2007). Quantum nature of a strongly coupled single quantum dot-cavity system. Nature, 445(7130), 896-899.

[9] Deng, H., Weihs, G., Santori, C., Bloch, J., \& Yamamoto, Y. (2002). Condensation of semiconductor microcavity exciton polaritons. Science, 298(5591), 199-202. 
[10] Kasprzak, J., Richard, M., Kundermann, S., Baas, A., Jeambrun, P., Keeling, J. M. J., Marchetti, F. M.,Szymanska, M. H., Andre,R., Staehli, J. L.,Savona,V., Littlewood, P. B., Deveaud,B.,

\& Dang, L. S. (2006). Bose-Einstein condensation of exciton polaritons. Nature, 443(7110), 409-414.

[11] McKeever, J., Boca, A., Boozer, A. D., Buck, J. R., \& Kimble, H. J. (2003). Experimental realization of a one-atom laser in the regime of strong coupling. Nature, 425(6955), 268-271.

[12] Chen, W., Beck, K. M., Bücker, R., Gullans, M., Lukin, M. D., Tanji-Suzuki, H., \& Vuletić, V. (2013). Alloptical switch and transistor gated by one stored photon. Science, 341(6147), 768-770.

[13] Volz, T., Reinhard, A., Winger, M., Badolato, A., Hennessy, K. J., Hu, E. L., \& Imamoğlu, A. (2012). Ultrafast all-optical switching by single photons. Nature Photonics, 6(9), 605-609.

[14] Wallraff, A., Schuster, D. I., Blais, A., Frunzio, L., Huang, R. S., Majer, J., Kumar, S., Girvin, S.M., \& Schoelkopf, R. J. (2004). Strong coupling of a single photon to a superconducting qubit using circuit quantum electrodynamics. Nature, 431(7005), 162-167.

[15] Balci, S., Kocabas, C., Ates, S., Karademir, E., Salihoglu, O., \& Aydinli, A. (2012). Tuning surface plasmon-exciton coupling via thickness dependent plasmon damping. Physical Review B, 86(23), 235402.

[16] Lakhtakia, A., \& Messier, R. (2005). Sculptured thin films: Nanoengineered morphology and optics (Vol. 143). SPIE press.

[17] Polo, J., Mackay, T., \& Lakhtakia, A. (2013). Electromagnetic surface waves: a modern perspective. Newnes.

[18] Hosseininezhad, S. H., \& Babaei, F. (2018). Excitation of multiple surface plasmon-polaritons by a metal layer inserted in an equichiral sculptured thin film. Plasmonics, 13(6), 1867-1879.

[19] Hosseininezhad, S. H., \& Babaei, F. (2019). On the propagation of multiple surface plasmon-polariton waves by a triangle helix thin film in sarid configuration. Plasmonics, 14(6), 1843-1854.

[20] Babaei, F., \& Rostami, M. (2019). Excitation of surface plexciton wave at interface of a metal and a columnar thin film infiltrated with J-aggregate dyes. Optics Communications, 439, 8-15.

[21] Babaei, F., \& Seyyedi, S. A. (2021). Excitation of Surface Plasmon-Polariton Wave at Both Interfaces of a Silver Thin Film in Two-Layer Kretschmann geometry. Plasmonics, 1-8.

[22] Gonçalves, P. A. D., Bertelsen, L. P., Xiao, S., \& Mortensen, N. A. (2018). Plasmon-exciton polaritons in two-dimensional semiconductor/metal interfaces. Physical Review B, 97(4), 041402.

[23] Wersall, M., Cuadra, J., Antosiewicz, T. J., Balci, S., \& Shegai, T. (2017). Observation of mode splitting in photoluminescence of individual plasmonic nanoparticles strongly coupled to molecular 
excitons. Nano letters, 17(1), 551-558.

[24] Zengin, G., Wersäll, M., Nilsson, S., Antosiewicz, T. J., Käll, M., \& Shegai, T. (2015). Realizing strong light-matter interactions between single-nanoparticle plasmons and molecular excitons at ambient conditions. Physical review letters, 114(15), 157401.

[25] Liu, W., Wang, Y., Naylor, C. H., Lee, B., Zheng, B., Liu, G., Charlie Johnson, A.T., Pan, A., \& Agarwal, R. (2018). Understanding the Different Exciton-Plasmon Coupling Regimes in Two-Dimensional Semiconductors Coupled with Plasmonic Lattices: A Combined Experimental and Unified Equation of Motion Approach. ACS Photonics, 5(1), 192-204.

\section{Figures}

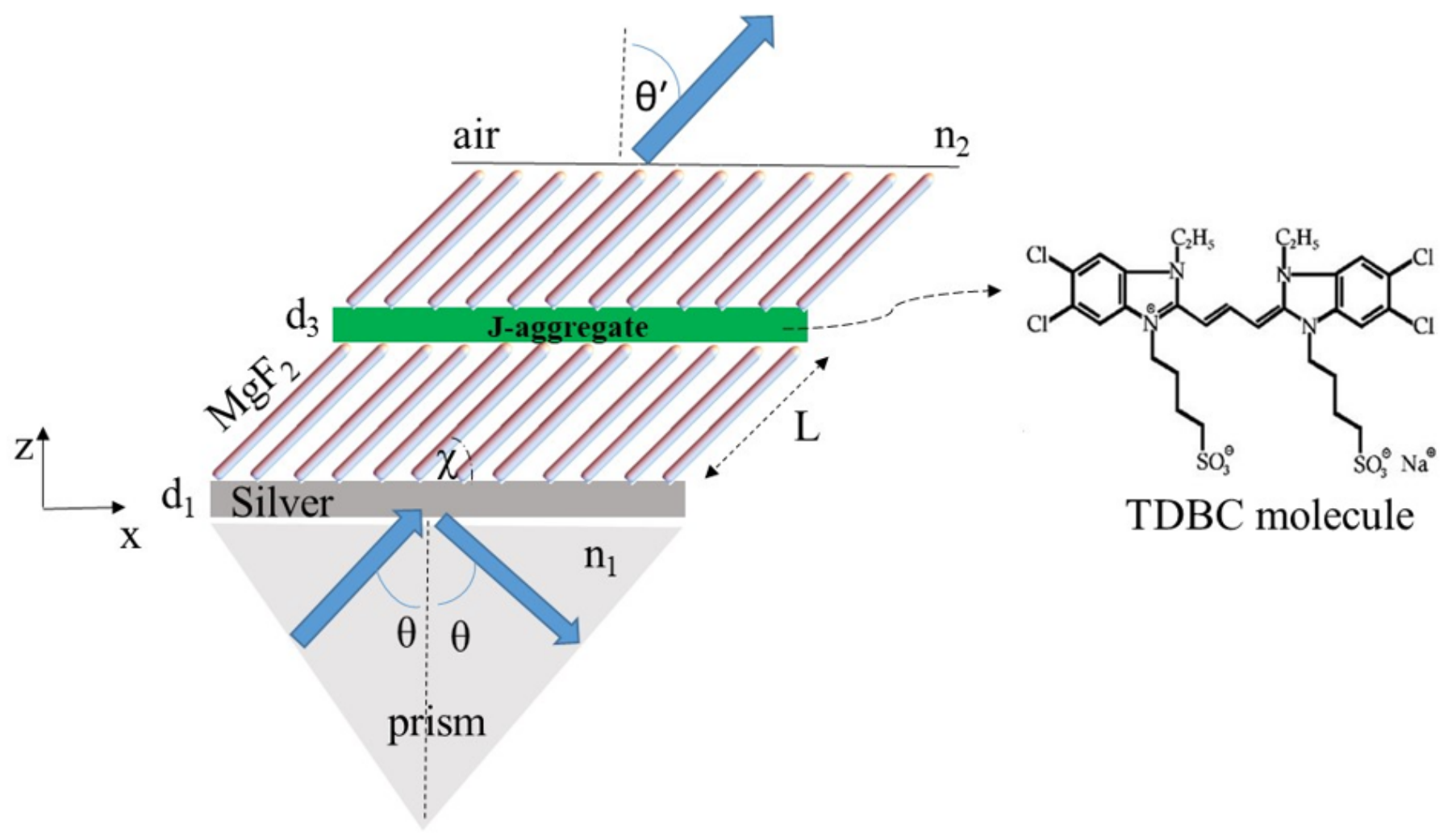

\section{Figure 1}

A schematic of columnar thin film with a central exciton slab in the Kretschmann configuration for coupling surface plasmon polariton and exciton. 

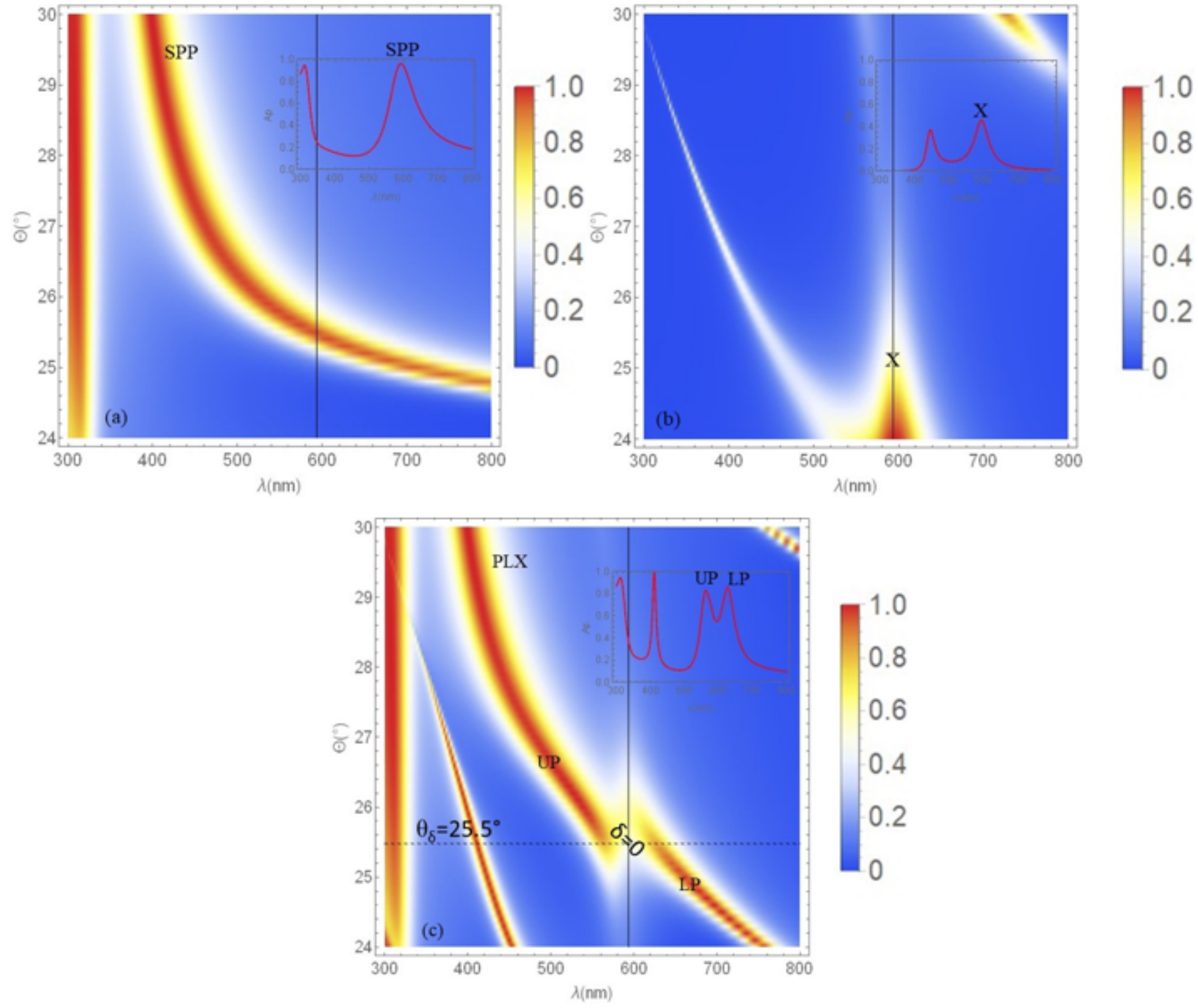

\section{Figure 2}

Absorption density plots as functions of wavelength and incident angle for excitation of (a) surface plasmon polariton(SPP), (b) surface exciton(X) and (c) surface plexciton(PLX). The zero detuning frequency is specified by a vertical line in each of plots. Inset plots show the depiction of optical absorption versus wavelength at $\theta \delta$ (where the detuning frequency is zero). In plexciton plot exist an anticrossing mode between LP and UP branches of polaritons at $\delta=0$ so that horizontal line shows the location of incident angle at zero detuning frequency. The used parameters were as: $d 1=40 \mathrm{~nm}, \mathrm{~L}=600$ $\mathrm{nm}, \mathrm{d} 3=286 \mathrm{~nm}, \mathrm{n} 1=2.57, \mathrm{\chi}=20^{\circ}, \mathrm{fv}=0.8, \theta \delta=25.5^{\circ}$. 

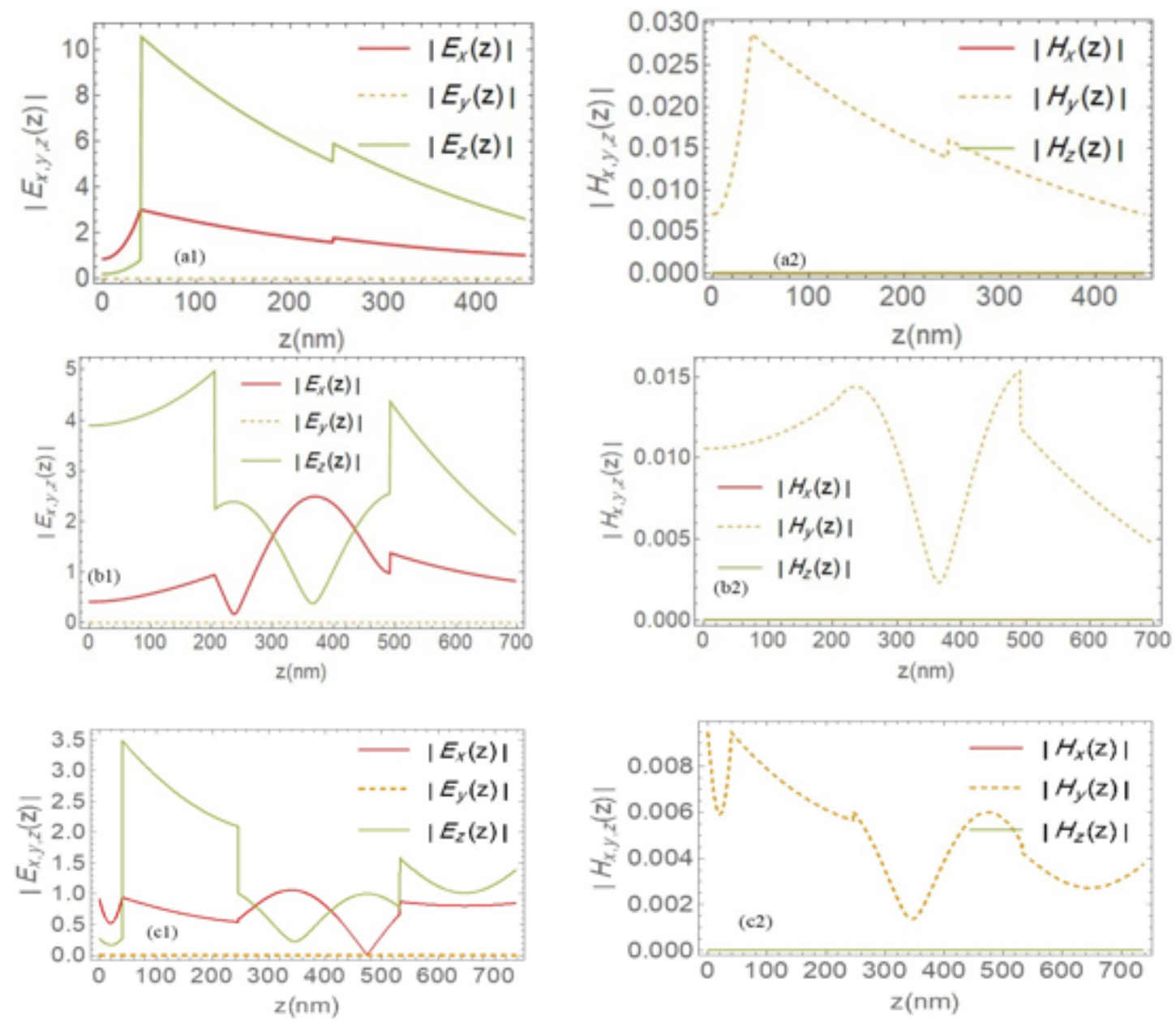

Fig.3. The plots show the absolute value of Cartesian components of electric $\left|E_{x, y, z}(z)\right|$ and magnetic fields $\left|H_{x, y, z}(z)\right|$ along the $\mathrm{z}$-axis at $\lambda=594 \mathrm{~nm}$ when a surface plasmon polariton wave excited(a1,b1), when a surface exciton wave excited(b1,b2) and when a surface plexciton wave excited(c1,c2). The computational parameters are same to Fig.2.

\section{Figure 3}

See image above for figure legend 

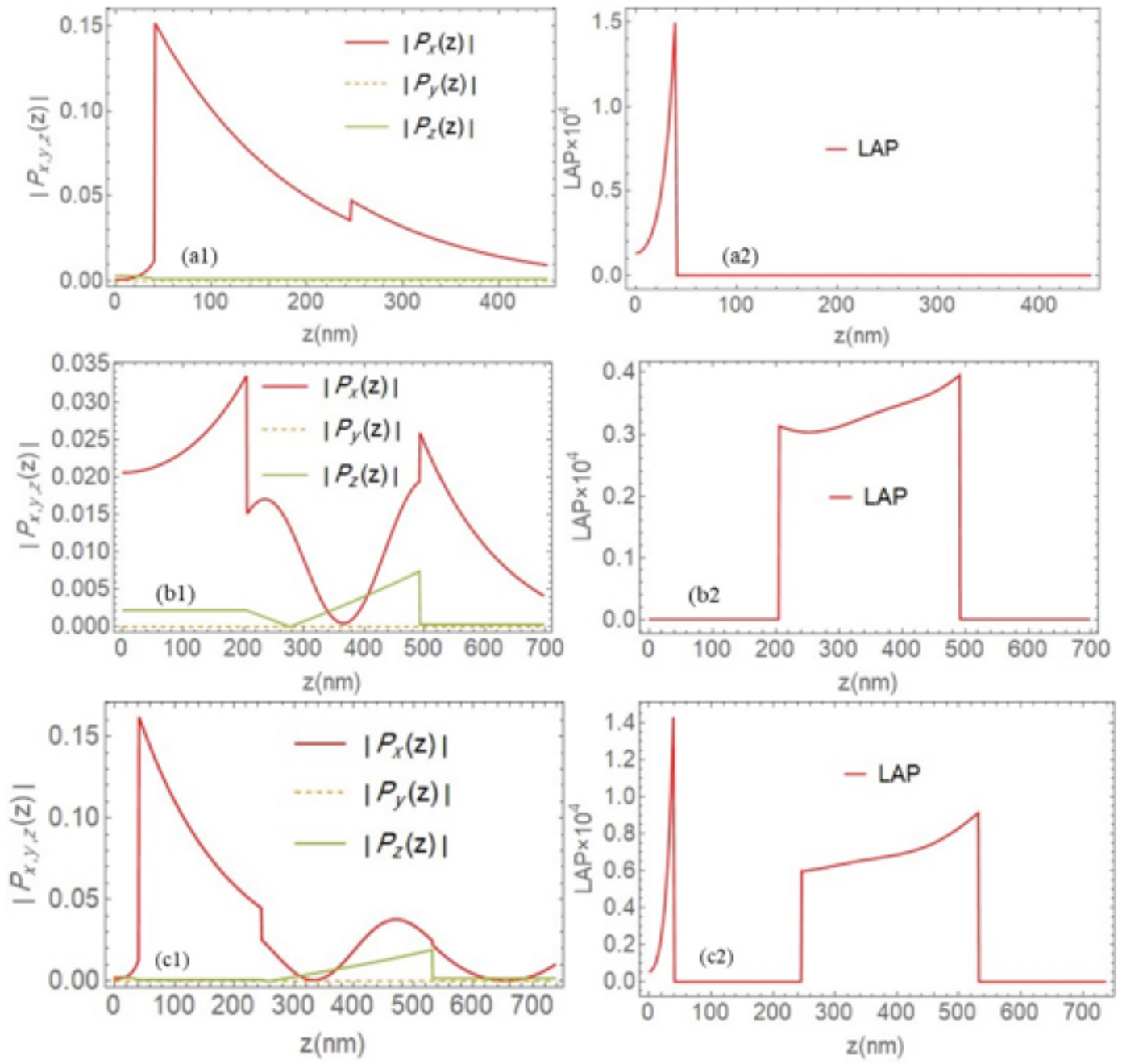

Fig.4.The plots of the Cartesian components of time-averaged Poynting vector $\left|p_{x, y, z}(z)\right|$ and local absorption (LAP) as a function of the depth of the structure.(a1,b1) propagation a surface plasmon polariton wave, $(\mathrm{b} 1, \mathrm{~b} 2)$ propagation a surface exciton wave and $(\mathrm{c} 1, \mathrm{c} 2)$ propagation a surface plexciton wave. For the used parameters, see Fig.3.

\section{Figure 4}

See image above for figure legend 

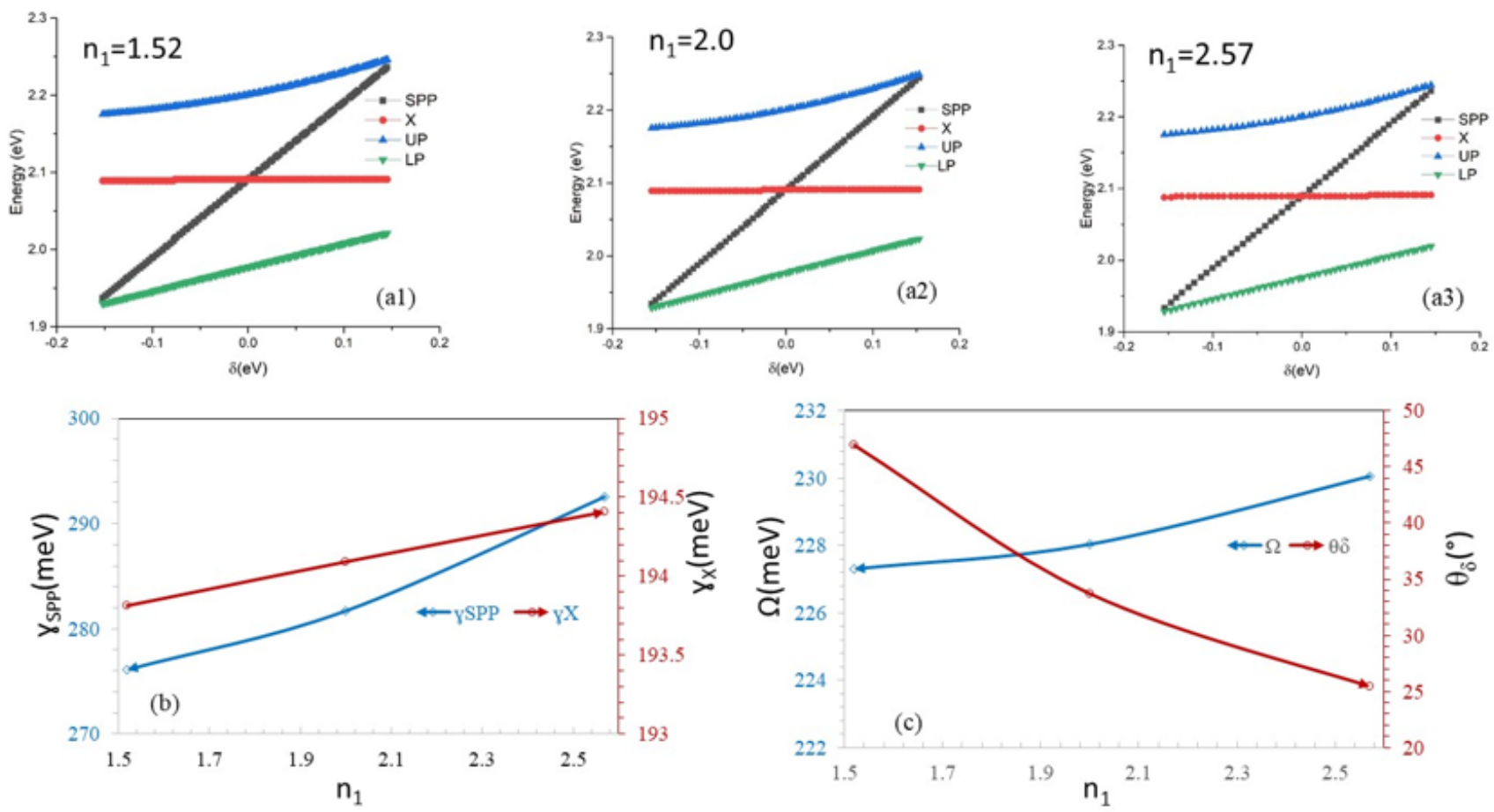

Fig.5. Energy of surface polariton modes as a function of detuning frequency (a1) $\mathrm{n} 1=1.52$, (a2) $\mathrm{n} 1=2.0$ and (a3) $\mathrm{n} 1=2.57$. The values of $\gamma_{S P P}$ and $\gamma_{X}$ for different refractive index of prism(n1) are shown in (b).Rabi splitting energy $\Omega$ and detuning angle $\theta_{\delta}$ as a function of refractive index of prism are given in (c). The configuration is described by the following parameters: $d_{1}=40 \mathrm{~nm}, \mathrm{~L}=600 \mathrm{~nm}$, $\mathrm{d}_{3}=286 \mathrm{~nm}, \chi=20^{\circ}, \mathrm{f}_{\mathrm{v}}=0.8$.

\section{Figure 5}

See image above for figure legend 

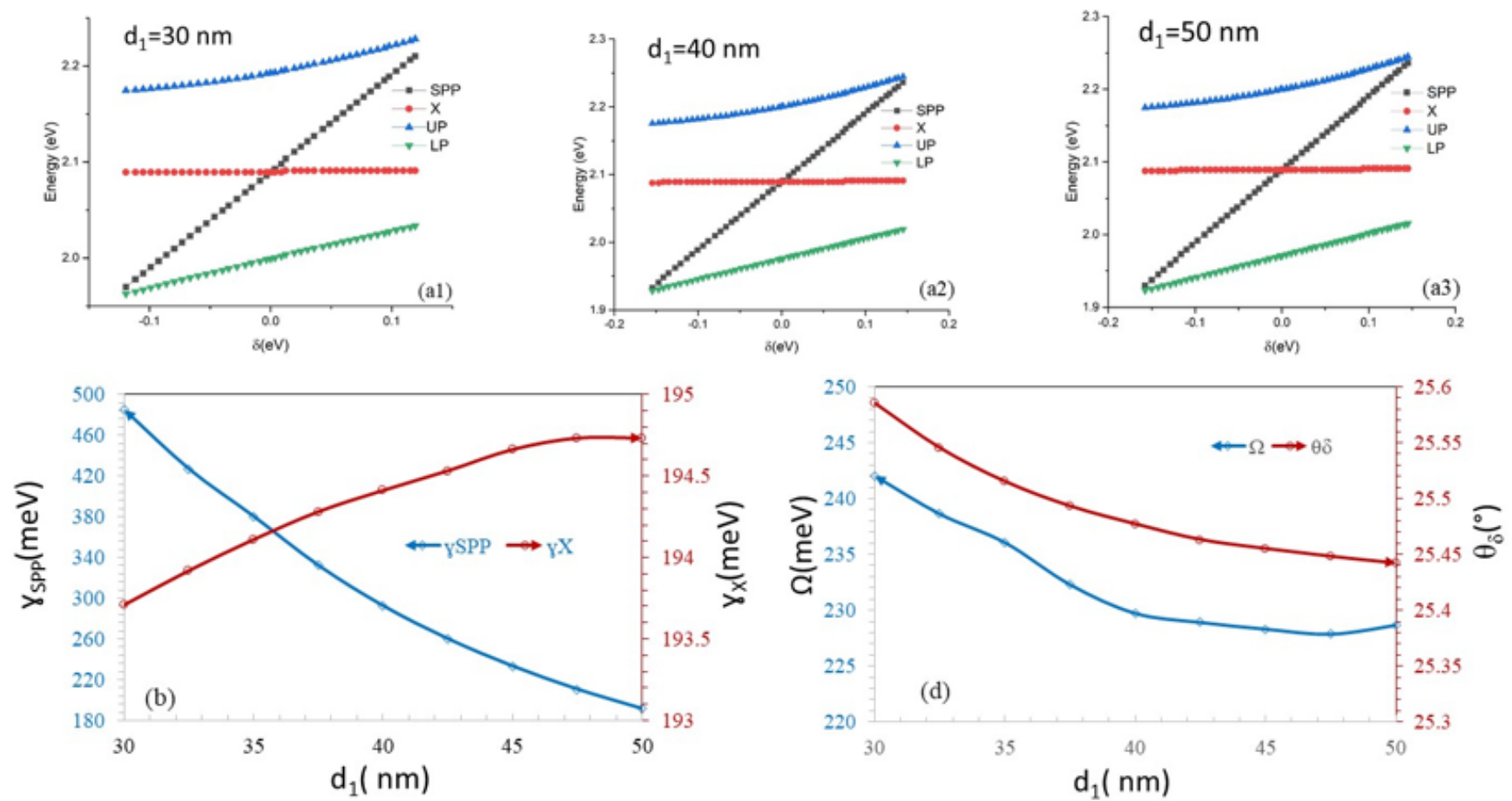

Fig.6. Energy of surface polariton modes as a function of detuning frequency (a1) $d_{1}=30 \mathrm{~nm}$, (a2) $\mathrm{d}_{1}=40 \mathrm{~nm}$ and (a3) $\mathrm{d}_{1}=50 \mathrm{~nm}$. The values of $\gamma_{S P P}$ and $\gamma_{X}$ for different thicknesses of metal $\left(\mathrm{d}_{1}\right)$ are shown in (b).Rabi splitting energy $\Omega$ and detuning angle $\theta_{\delta}$ as a function of thickness of metal are given in (c). The configuration is described by the following parameters: $L=600 \mathrm{~nm}, \mathrm{~d}_{3}=286 \mathrm{~nm}$, $\mathrm{n}_{1}=2.57, \chi=20^{\circ}, \mathrm{f}_{\mathrm{V}}=0.8$.

\section{Figure 6}

See image above for figure legend 

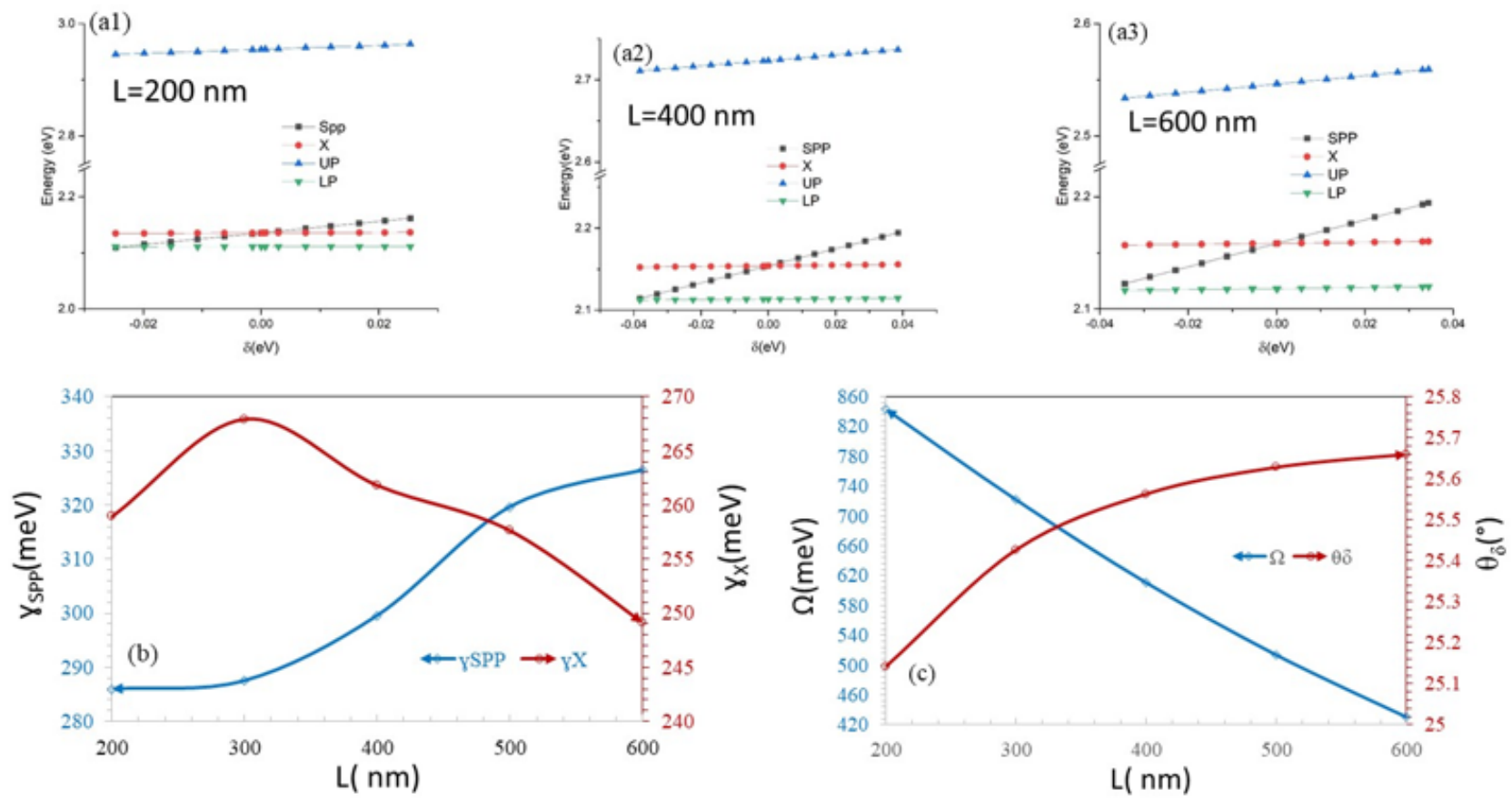

Fig.7. Energy of surface polariton modes as a function of detuning frequency (a1) $L=200 \mathrm{~nm}$, (a2) $\mathrm{L}=400 \mathrm{~nm}$ and (a3) $\mathrm{L}=600 \mathrm{~nm}$. The values of $\gamma_{S P P}$ and $\gamma_{X}$ for different lengths of nanocolumns of dielectric medium(L) are shown in (b).Rabi splitting energy $\Omega$ and detuning angle $\theta_{\delta}$ as a function of $\mathrm{L}$ are given in (c). The configuration is described by the following parameters: $d_{1}=40 \mathrm{~nm}, \mathrm{~d}_{3}=150$ $\mathrm{nm}, \mathrm{n}_{1}=2.57, \chi=25^{\circ}, \mathrm{f}_{\mathrm{v}}=0.8$.

\section{Figure 7}

See image above for figure legend 

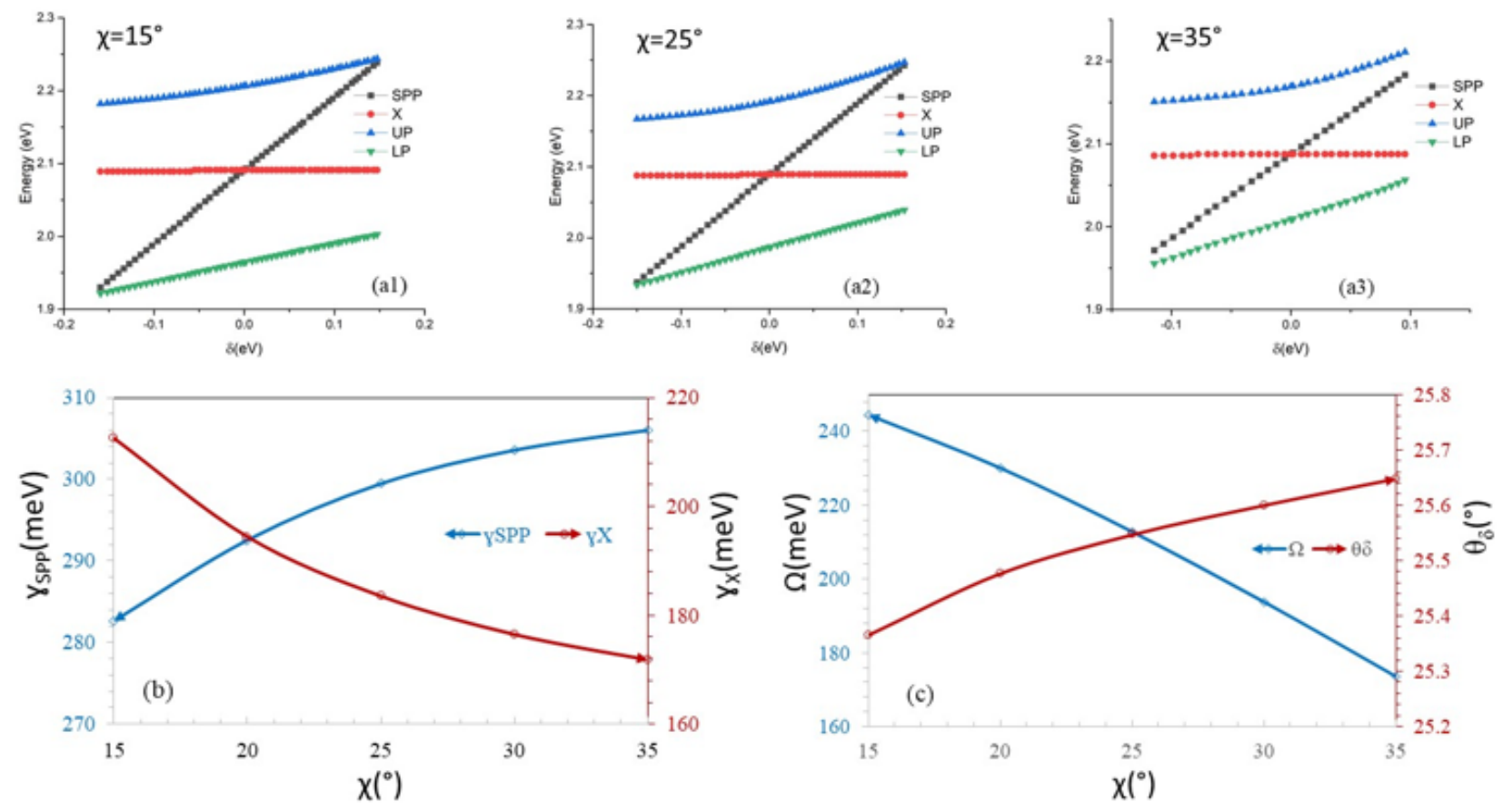

Fig.8. Energy of surface polariton modes as a function of detuning frequency (a1) $\chi=15^{\circ}$, (a2) $\chi=25^{\circ}$ and (a3) $\chi=35^{\circ}$. The values of $\gamma_{S P P}$ and $\gamma_{X}$ for different tilt angles of nanocolumns of dielectric medium $(\chi)$ are shown in (b).Rabi splitting energy $\Omega$ and detuning angle $\theta_{\delta}$ as a function of $\chi$ are given in (c). The configuration is described by the following parameters: $d_{1}=40 \mathrm{~nm}, \mathrm{~L}=600 \mathrm{~nm}, \mathrm{~d}_{3}=$ $286 \mathrm{~nm}, \mathrm{n}_{1}=2.57, \mathrm{f}_{\mathrm{v}}=0.8$.

\section{Figure 8}

See image above for figure legend 

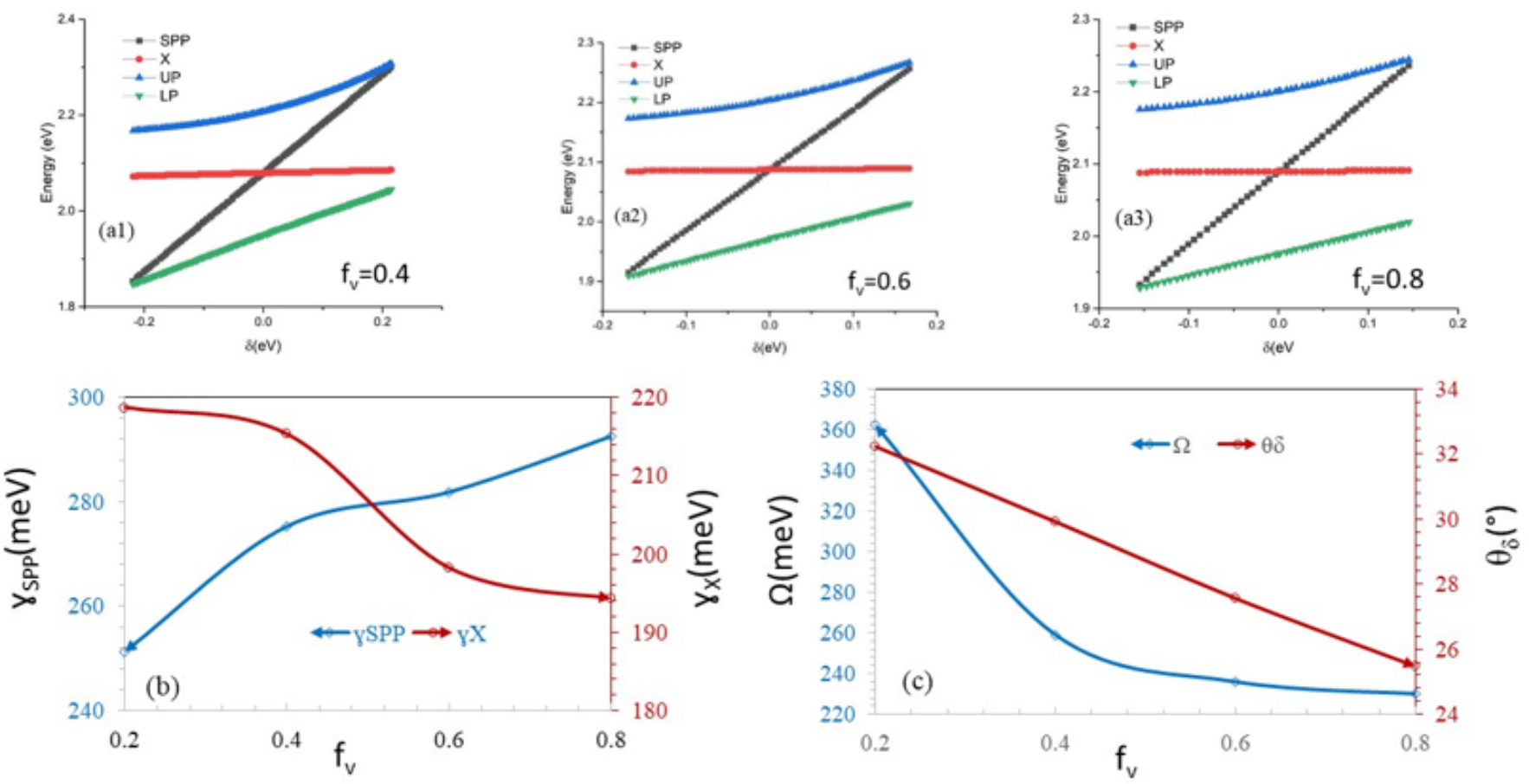

Fig.9. Energy of surface polariton modes as a function of detuning frequency (a1) $f_{v}=0.4$, (a2) $f_{v}$ $=0.6$ and $(\mathrm{a} 3) \mathrm{f}_{\mathrm{v}}=0.8$. The values of $\gamma_{S P P}$ and $\gamma_{X}$ for different void fractions of dielectric medium $\left(\mathrm{f}_{\mathrm{v}}\right)$ are shown in (b).Rabi splitting energy $\Omega$ and detuning angle $\theta_{\delta}$ as a function of $f_{v}$ are given in (c). The configuration is described by the following parameters: $d_{1}=40 \mathrm{~nm}, \mathrm{~L}=600 \mathrm{~nm}, \mathrm{n}_{1}=2.57$. The selected of thickness of excitonic slab and tilt angle of nanocolumns of dielectric medium at void fractions of dielectric $0.2,0.4,0.6$ and 0.8 were respectively as: $15^{\circ}, 478 \mathrm{~nm} ; 20^{\circ}, 370 \mathrm{~nm} ; 20^{\circ}, 315 \mathrm{~nm}$ and $20^{\circ}, 286 \mathrm{~nm}$.

\section{Figure 9}

See image above for figure legend 

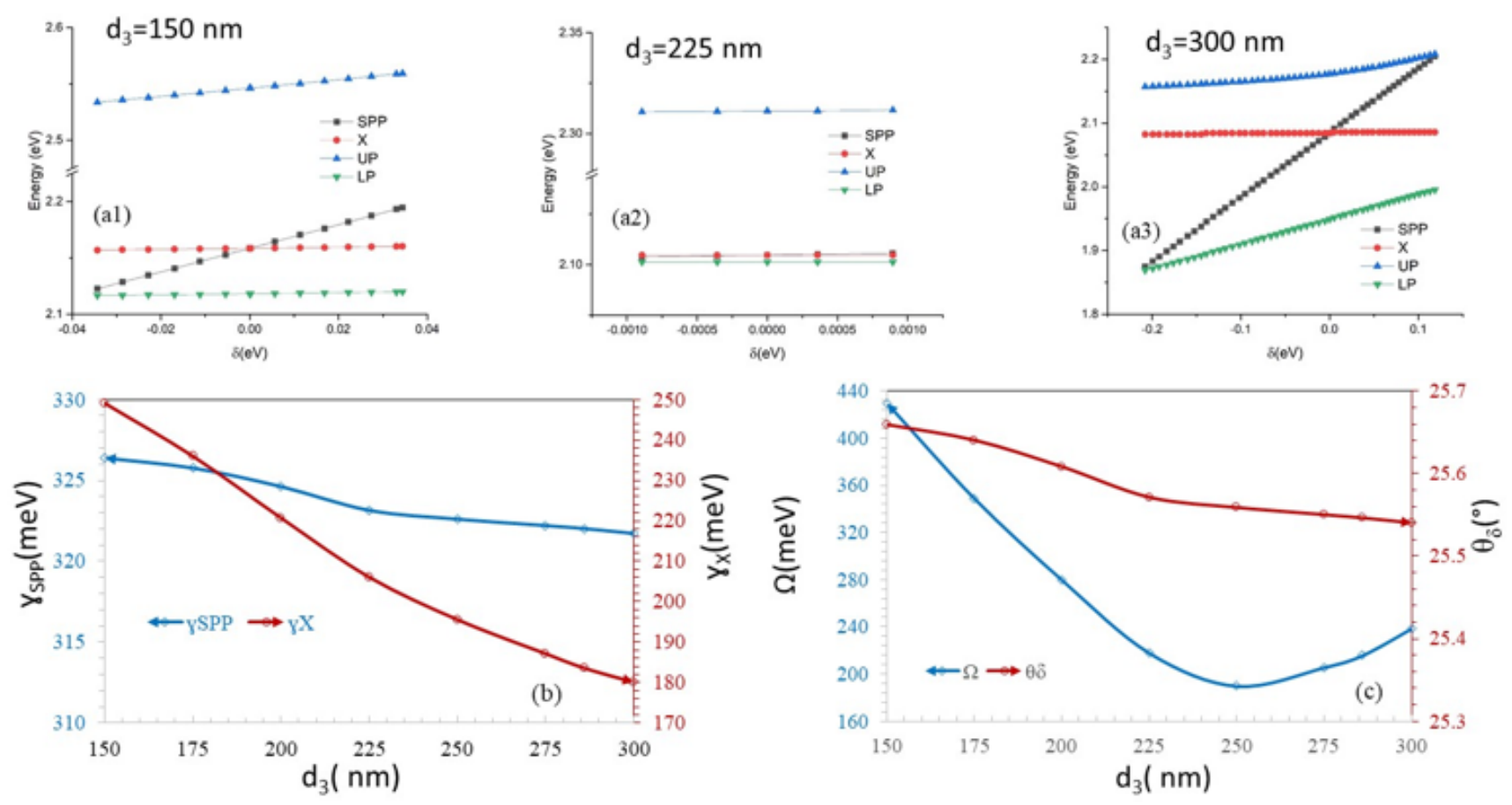

Fig.10. Energy of surface polariton modes as a function of detuning frequency (a1) $d_{3}=150 \mathrm{~nm}$, (a2) $\mathrm{d}_{3}=225 \mathrm{~nm}$ and (a3) $\mathrm{d}_{3}=300 \mathrm{~nm}$. The values of $\gamma_{S P P}$ and $\gamma_{X}$ for different thicknesses of exciton slab $\left(d_{3}\right)$ are shown in (b).Rabi splitting energy $\Omega$ and detuning angle $\theta_{\delta}$ as a function of $d_{3}$ are given in (c). The configuration is described by the following parameters: $d_{1}=40 \mathrm{~nm}, \mathrm{~L}=600 \mathrm{~nm}, \chi=25^{\circ}$, $\mathrm{n}_{1}=2.57, \mathrm{f}_{\mathrm{v}}=0.8$.

\section{Figure 10}

See image above for figure legend 